\title{
Uma Abordagem Evolutiva para o Problema de Sequenciamento Curricular Adaptativo
}

\author{
Marcelo O. C. Machado ${ }^{1}$, Eduardo Barrére ${ }^{1}$, Jairo F. Souza ${ }^{1}$ \\ ${ }^{1}$ Departamento de Ciência da Computação - \\ Universidade Federal de Juiz de Fora (UFJF) \\ 36.360-900 - Juiz de Fora - MG - Brasil \\ \{marcelomachado, eduardo.barrere, jairo.souza\}@ice.ufjf.br
}

\begin{abstract}
Adaptive Curriculum Sequencing (ACS) is still a challenge in Adaptive Learning field. ACS is a NP-Hard problem especially considering the constraints of the student and learning materials when selecting a sequence from repositories where several sequences could be chosen. Therefore, this has stimulated researchers to use evolutionary approaches in the search for satisfactory solutions. This work uses the Prey-Predator Algorithm, which have shown to be suitable for the problem, according to laboratory experiments, although it is a solution not yet explored in the Adaptive Learning literature.
\end{abstract}

Resumo. O Sequenciamento Curricular Adaptativo (SCA) ainda é um desafio na área de Aprendizagem Adaptativa. O SCA é um problema NP-Difícil, principalmente por considerar as restrições relacionadas ao perfil do aluno e dos materiais didáticos na seleção de uma sequência em repositórios onde várias poderiam ser escolhidas. Portanto, isso tem estimulado pesquisadores a utilizarem abordagens evolutivas na busca por soluções satisfatórias. Este trabalho utiliza o Algoritmo Presa-Predador, o qual se mostrou adequado para o problema, de acordo com experimentos realizados em laboratório, embora seja uma solução ainda não explorada na literatura de Aprendizagem Adaptativa.

\section{Introdução}

A efetiva utilização de sistemas de aprendizagem on-line depende da criação de soluções para correta administração dos recursos educacionais [Van Merriënboer and Ayres 2005]. A maioria dos cursos ofertados em Ambientes Virtuais de Aprendizagem (AVA) tradicionais e Massive Open Online Courses (MOOCs) ainda são baseados na forma passiva de apresentação do conteúdo aos alunos [Acampora et al. 2011], esse fator atrelado a omissões relevantes, tal como a contextualização da experiência de aprendizagem do aluno, são considerados os principais obstáculos à adoção desses sistemas [Acampora et al. 2011].

Sistemas de e-learning adaptativo, através da integração com Sistemas Tutores Inteligentes (STIs), são considerados como alternativa para abordar alguns dos problemas supracitados, pois capturam informações do aluno com o objetivo de personalizar a instrução [Phobun and Vicheanpanya 2010]. A adaptação está em vários âmbitos, por exemplo, em nível de sequenciamento curricular [Premlatha and Geetha 2015], cujo intuito é auxiliar o sistema a encontrar a melhor sequência de recursos educacionais que 
VII Congresso Brasileiro de Informática na Educação (CBIE 2018)

Anais do XXIX Simpósio Brasileiro de Informática na Educação (SBIE 2018)

satisfaçam os objetivos, características e preferências de aprendizagem do aluno. Portanto, o desafio está na maneira que o sequenciamento é criado, pois caso seja feito de maneira inadequada pode levar a consequências negativas, como a falta de interesse do aluno pelo curso e consequentemente a evasão [Xie et al. 2017].

A partir do acesso facilitado às tecnologias de criação e disponibilização de conteúdo e do movimento de Recursos Educacionais Abertos (REAs) [Butcher 2015], os repositórios utilizados por sistemas de e-learning ficam cada vez maiores. Considerando as diversas restrições dos alunos e dos recursos educacionais, encontrar o sequenciamento curricular mais adequado ao aluno, a partir desses repositórios, se apresenta como um problema de otimização combinatória pertencente a classe de problemas NPDifícil [Pushpa 2012]. Para abordar esse problema - nomeado Sequenciamento Curricular Adaptativo (SCA) - este trabalho apresenta uma adaptação do Algoritmo PresaPredador (APP) [Tilahun and Ong 2015] a qual demonstrou, a partir de experimentos, ser uma meta-heurística adequada para tratar o problema embora seja uma solução ainda não explorada pela literatura.

O restante do artigo está organizado da seguinte forma: a Seção 2 formaliza o problema de SCA; a Seção 3 apresenta os trabalhos relacionados; a Seção 4 apresenta a proposta do trabalho e, por fim, a Seção 5 apresenta as conclusões do trabalho.

\section{Sequenciamento Curricular Adaptativo}

A pesquisa e desenvolvimento de STIs busca unir técnicas de Inteligência Artificial, Psicologia Cognitiva e Teorias de Aprendizagem Educacional com o objetivo de criar sistemas capazes de saber o que ensina, a quem ensina e como ensina [Nwana 1990, Silva et al. 2018]. Nesse cenário, o problema de SCA se mostra como uma pesquisa crucial na área de Aprendizagem Adaptativa [Muhammad et al. 2016].

De acordo com [Brusilovsky 2003], o objetivo do SCA é fornecer ao aluno a ordenação mais adequada de unidades e tarefas de conhecimento (exemplos, perguntas, problemas, etc). A intenção é ajudar o aluno a encontrar um "caminho ideal" de aprendizagem dentro do domínio de conhecimento [Hafidi and Bensebaa 2015], maximizando a compreensão, bem como a eficiência na aprendizagem. Segundo [Kardan et al. 2015], o SCA deve levar em consideração as características dos alunos e as informações dos materiais didáticos. Portanto, o SCA pode ser visto como uma função $f(a, d, e) \rightarrow S$ que recebe como parâmetros o modelo do aluno (representação do aluno a partir dos seus dados) $a$, as informações dos materiais didáticos $d$ e as informações da estrutura pedagógica de conceitos $e$ contendo as interligações entre os conceitos abordados em um curso. Essa função tem como retorno uma sequência $s \in S$ que melhor se aproxima do modelo do aluno dentre as diversas possibilidades de sequências contidas em $S$.

Encontrar o melhor SCA é um problema da classe NP-Difícil [Pushpa 2012], visto que os repositórios utilizados pelos sistemas são constantemente atualizados à medida que novos recursos educacionais são adicionados. Nesse cenário, considere um curso que aborda um conjunto $C$ de conceitos de aprendizagem e que cada um desses conceitos pode estar relacionado a diversos materiais didáticos. Então, o número de possíveis sequências seria equivalente à multiplicação entre o número de materiais didáticos de cada um desses conceitos. Portanto, se cada conceito é abordado igualmente por um conjunto $M$ de materiais didáticos, tem-se $|M|^{|C|}$ possibilidades de sequências que poderiam ser seleci- 
VII Congresso Brasileiro de Informática na Educação (CBIE 2018)

Anais do XXIX Simpósio Brasileiro de Informática na Educação (SBIE 2018)

onadas. O problema se agrava caso sejam consideradas permutações entre os conceitos, subsequências e, sobretudo, restrições em relação às necessidades dos alunos.

\section{Trabalhos Relacionados}

Por se tratar de um problema da classe NP-Difícil, o SCA motivou diversos pesquisadores a utilizarem heurísticas e meta-heurísticas como solução do problema, pois classicamente, elas apresentaram-se como boas alternativas a problemas da mesma natureza [Al-Muhaideb and Menai 2011, Machado et al. 2017].

[Al-Muhaideb and Menai 2011] apresentam uma revisão da literatura relacionada aos trabalhos que utilizaram abordagens evolutivas para o problema de SCA, bem como [Machado et al. 2017] propõem um modelo de comparação de trabalhos, da mesma área, a partir dos parâmetros utilizados no sequenciamento. Os trabalhos revisados nessas duas pesquisas se relacionam à proposta deste trabalho. Entretanto, algumas dessas abordagens apresentam maior semelhança e, portanto necessitam destaque: no trabalho de [Li et al. 2012] são comparadas soluções que usam Algoritmo Genético (AG) e Otimização por Enxame de Partículas (OEP) para o SCA. A função objetivo utilizada foi composta pela agregação de múltiplos objetivos visando atender as necessidades do aluno. O presente trabalho apresenta adaptações no cálculo de alguns objetivos propostos em [Li et al. 2012] (Eq. 7, 8 e 9); em [Hnida et al. 2016] é aplicado um Algoritmo de Busca Harmônica, onde a função objetivo se baseia no conhecimento prévio do aluno e na relação entre os conceitos do curso.

Este trabalho se diferencia dos trabalhos anteriormente citados nos seguintes aspectos: primeiramente, são considerados diversos parâmetros extrínsecos e intrínsecos do aluno na função objetivo, além das informações dos materiais e suas relações (Seção 4). Assim, o conjunto de variáveis para o problema é maior, o que permite uma melhor adaptação da sequência. Além disso, é adotada uma meta-heurística ainda não explorada pela literatura, cuja motivação de utilização se deu a partir de experimentos demonstrados em [Tilahun and Ong 2015] onde o APP apresentou melhores resultados que AG e OEP em cinco problemas clássicos de otimização. Esse fato colabora com a hipótese de que o APP pode ser uma alternativa plausível para o problema de SCA, visto que AG e OEP são meta-heurísticas largamente utilizadas neste campo de pesquisa [Al-Muhaideb and Menai 2011, Machado et al. 2017].

\section{O Algoritmo Presa-Predador para o problema de Sequenciamento Curricular Adaptativo}

O Algoritmo Presa-Predador é inspirado na interação ecológica entre organismos vivos que se alimentam de outras espécies [Tilahun and Ong 2015]. No contexto deste trabalho, cada indivíduo (animal) representa uma solução, ou seja, uma sequência de materiais didáticos selecionados a partir de um repositório. O indivíduo foi modelado como um vetor de números binários $s=\left\{x_{1}, x_{2}, \ldots, x_{|M|-1}, x_{|M|}\right\}$, onde $M$ representa o conjunto de materiais no repositório e $x_{i}$ denota um valor binário. Cada material didático está relacionado a uma posição do vetor, onde o valor binário 1 na posição indica que aquele material faz parte da sequência e o valor 0 indica que o material não faz parte da sequência. No decorrer das iterações do algoritmo, os valores são alterados dentro das sequências do espaço de solução. Ao final de cada iteração, as sequências são avaliadas com o objetivo de verificar a adequação ao perfil do aluno alvo. 
VII Congresso Brasileiro de Informática na Educação (CBIE 2018)

Anais do XXIX Simpósio Brasileiro de Informática na Educação (SBIE 2018)

Cada um dos indivíduos $s \in S$ é associado a um valor calculado a partir da função objetivo $C M(s)$. A função está relacionada à otimização do problema. Neste trabalho, a otimização está ligada a um problema de minimização, isto é $\min _{s \in S} C M(s)$. Considerando um conjunto $S$ de soluções candidatas, $s_{j}$ é dita como solução ótima global para o problema de minimização se $s_{j} \in S$ tal que $C M\left(s_{j}\right) \leq C M\left(s_{i}\right), \forall s_{i} \in S$.

Inicialmente, os valores gerados pela função são associados aos indivíduos e indica a sua chance de morte ${ }^{1}$. Em seguida, esses indivíduos são declarados como presas ou predador, onde o indivíduo com maior chance de morte é considerado o predador (caso ele não se alimente logo irá morrer) e as demais soluções candidatas são consideradas presas. A presa que tem o menor chance de morte recebe o rótulo de melhor presa e as demais recebem o rótulo de presa comum.

A exploração do espaço de busca é feita pela interação entre as presas e o predador. Enquanto o predador está caçando as presas e elas estão fugindo, tem-se a fase de diversificação da busca. A fase de intensificação da busca se dá pela melhor presa, a qual realiza uma busca local pela vizinhança de forma a encontrar uma posição que diminua sua chance de morte. A analogia indica que a melhor presa tem menor chance de morte, pois ela encontrou um esconderijo e, assim, não é afetada pelo predador.

Dois fatores básicos são levados em consideração sobre o movimento dos indivíduos: (1) a direção na qual o movimento ocorrerá e (2) o comprimento do passo, que indica quanto a presa ou predador irá caminhar em uma direção. Neste trabalho, ao dizer que um indivíduo está caminhando em direção a outro, entende-se que suas representações binárias estão se tornando mais semelhante. Ou seja, indivíduos que estão no mesmo local são exatamente iguais e, portanto, apresentam a mesma representação. Para o cálculo da distância entre dois indivíduos é utilizada a distância de Hamming. Quanto maior o valor retornado pela distância de Hamming, mais distante um indivíduo está do outro.

A direção na qual uma presa comum irá se movimentar pode levar em consideração a localização das presas com menor chance de morte. Nesse caso, é necessário identificar essas presas $\left\{s_{1}, s_{2}, \ldots, s_{p}\right\}$. As presas tendem a se reunir com presas que estão mais próximas a ela, ou seja, não apenas a chance de morte é considerada para a direção, mas também a distância. Dada uma presa $s_{i}$, para cada presa $s_{j}$, com $i \neq j \mid C M\left(s_{j}\right)<C M\left(s_{i}\right)$, é calculado $C S\left(s_{j}\right) \in[0,1]$, a qual indica a chance daquela presa ser seguida, segundo a Eq. 1, onde $\tau \in[0,1]$ representa o peso da distância para o movimento, $\eta \in[0,1]$ representa o peso da chance de morte para o movimento, $H\left(s_{j}, s_{i}\right)$ é a distância de Hamming entre os indivíduos, $m$ indica a quantidade de dimensões dos vetores que representam os indivíduos e $C M$ é a função objetivo que indica a chance de morte. A partir desses valores, é utilizada a técnica da roleta, a qual será girada para cada passo de uma presa comum, indicando qual presa deverá ser seguida. A chance de uma presa $s_{j}$ ser selecionada pela roleta é proporcional ao valor de $C S\left(s_{j}\right)$.

$$
C S\left(s_{j}\right)=1-\frac{\tau \frac{H\left(s_{j}, s_{i}\right)}{m}+\eta \frac{C M\left(s_{j}\right)}{C M\left(s_{i}\right)}}{2}
$$

\footnotetext{
${ }^{1}$ No trabalho de [Tilahun and Ong 2015] o termo utilizado é Valor de Sobrevivência, todavia o contexto está relacionado a um problema de maximização. No presente artigo é utilizado o termo de Chance de Morte para manter a relação diretamente proporcional com um problema de minimização.
} 
VII Congresso Brasileiro de Informática na Educação (CBIE 2018)

Anais do XXIX Simpósio Brasileiro de Informática na Educação (SBIE 2018)

Para cálculo da quantidade de passos que $s_{i}$ irá dar em direção às melhores presas, é preciso considerar sua distância com relação ao predador, visto que, quanto mais próximo, mais rápido a presa deve se movimentar e vice-versa. A quantidade de passos $l_{\text {max }}(i)$ é dada pela Eq. 2. O parâmetro $\beta \in[0,1]$ é um peso que representa a importância da distância entre a presa e o predador para o cálculo. Além disso, $\lambda_{\max } \leq m$ é um parâmetro que indica a quantidade máxima de passos que pode ser dada por qualquer indivíduo do conjunto de soluções e $\varepsilon \in[0,1]$ é gerado aleatoriamente durante a interação.

$$
l_{\text {max }}(i)=\frac{\lambda_{\text {max }} \varepsilon}{e^{\beta \frac{H\left(s_{i}, s_{\text {predador }}\right)}{m}}}
$$

Aleatoriamente, uma presa pode fugir do predador ao invés de seguir as demais presas. Nesse caso, é sorteada uma direção aleatória $y_{r}$ que é comparada com sua direção complementar $\overline{y_{r}}$ a fim de verificar qual direção deixaria a presa em posição mais distante do predador (Eq. 3 e 4 ). Se $d 1>d 2$, então $y_{r}$ será considerada a direção aleatória na qual a presa irá fugir. Do contrário, $\overline{y_{r}}$ será a direção escolhida.

$$
\begin{aligned}
& d_{1}=H\left(s_{\text {predador }}, y_{r}\right) \\
& d_{2}=H\left(s_{\text {predador }}, \overline{y_{r}}\right)
\end{aligned}
$$

Por fim, o predador irá seguir a presa com maior chance de morte com uma quantidade de passos $l_{\text {predador }}=\lambda_{\min } \varepsilon$, onde $\lambda_{\min } \leq \lambda_{\max } \leq m$ é um parâmetro que indica a quantidade mínima de passos que pode ser dada por qualquer indivíduo.

Para todos os casos de movimentação, anteriormente descritos, é considerada uma direção aleatória $y_{r}$ que colabora com a diversificação.

O número de iterações foi definido como critério de parada nos experimentos deste trabalho e a cada iteração os indivíduos são atualizados até que o critério seja atendido. Os passos do APP estão resumidos no fluxograma da Figura 1.

A função objetivo é dada pela Equação 5, onde $\omega_{i}$ corresponde ao respectivo peso que pode ser associado a cada uma das cinco funções da Tabela 1.

$$
f=\sum_{i=1}^{5} \omega_{i} O_{i}
$$

Nesta tabela, $x_{i}$ indica um valor binário da i-ésima posição de um vetor solução $s \in S$, $m_{i} \in M$ representa o i-ésimo material didático, $c_{j} \in C$ representa o j-ésimo conceito de um curso. Com relação ao aluno $a_{k}$, são considerados os seguintes parâmetros: o grau de habilidade $H_{c_{j}}^{a_{k}}$ em relação a um conceito $c_{j}$, a indicação $E_{c_{j}}^{a_{k}}$ se o conceito $c_{j}$ é esperado pelo aluno, onde $E_{c_{j}}^{a_{k}}=1$ se o conceito $c_{j}$ deve ser entregue ao aluno e $E_{c_{j}}^{a_{k}}=0$ caso contrário, os limites inferior $T_{\text {inf }}^{a_{k}}$ e superior $T_{\text {sup }}^{a_{k}}$ de tempos esperados para duração do curso, o conjunto de conceitos $E^{a_{k}}$ que fazem parte dos objetivos de aprendizagem do aluno e $\theta_{f}^{m_{i}}$ que indica quais os Estilos de Aprendizagem (EA) de cada uma das 4 dimensões $l$ do Felder and Silverman Learning Style Model (FSLSM) [Felder et al. 1988] o material tende a contemplar com mais intensidade - o FSLSM foi utilizado por ser considerado um dos mais utilizados na área [do Nascimento et al. 2017]. Com relação a cada 
VII Congresso Brasileiro de Informática na Educação (CBIE 2018)

Anais do XXIX Simpósio Brasileiro de Informática na Educação (SBIE 2018)

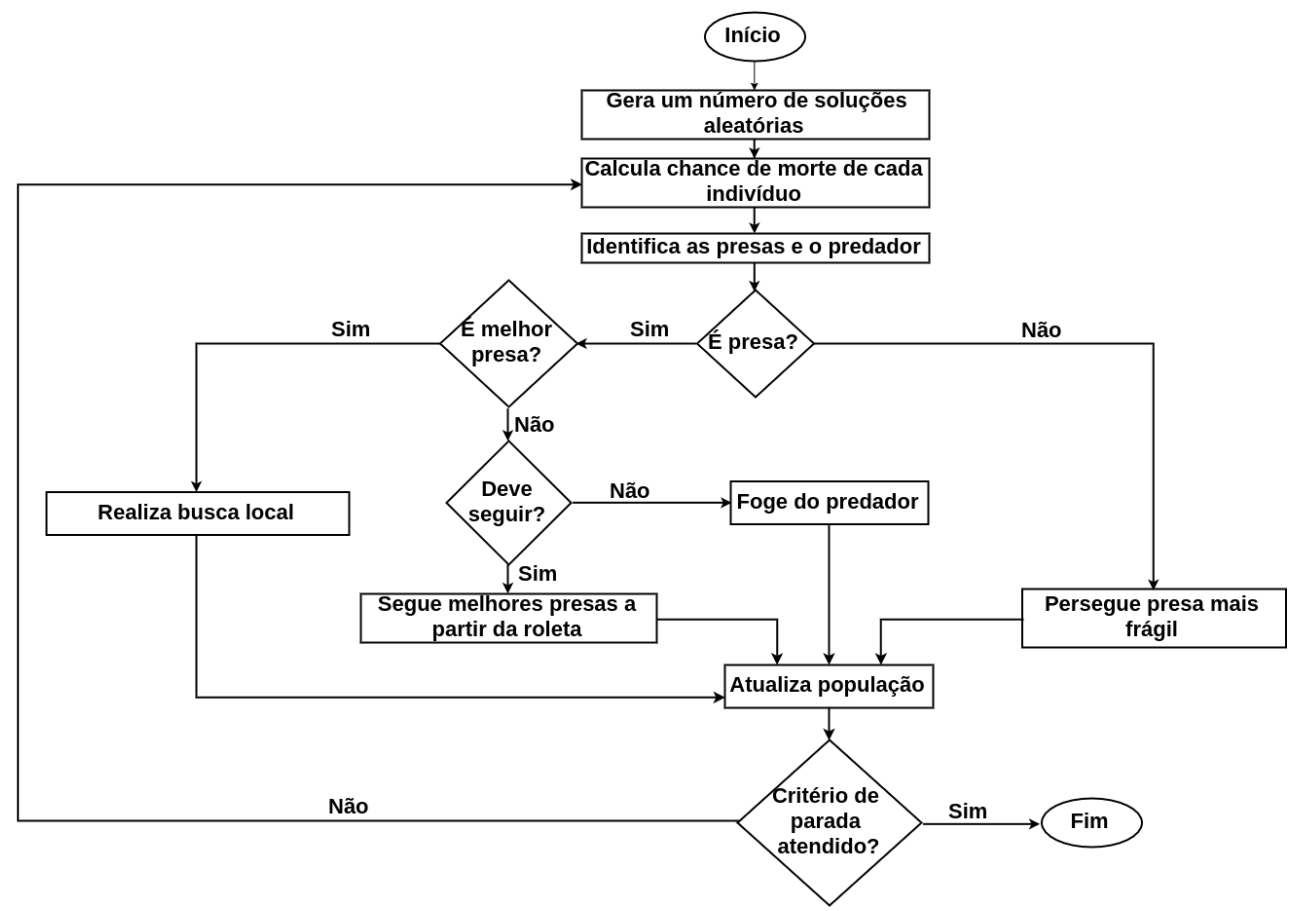

Figura 1. Fluxograma de execução do Algoritmo Presa-Predador.

um dos materiais didático $m_{i}$ são considerados os seguintes parâmetros: o grau de dificuldade $D^{m_{i}}$, a indicação $R_{c_{j}}^{m_{i}}$ se o material didático aborda um conceito $c_{j}$ onde $R_{c_{j}}^{m_{i}}=1$ se o material didático aborda o conceito $c_{j}$ e $R_{m_{i}}^{c_{j}}=0$ caso contrário, o tempo estimado de aprendizagem $T_{e s t}^{m_{i}}$, o conjunto de conceitos $R^{m_{i}}$ que o material didático aborda e $L_{f}^{a_{k}}$ que indica quais EA das dimensões $l$ do FSLSM o aluno tem mais intensidade.

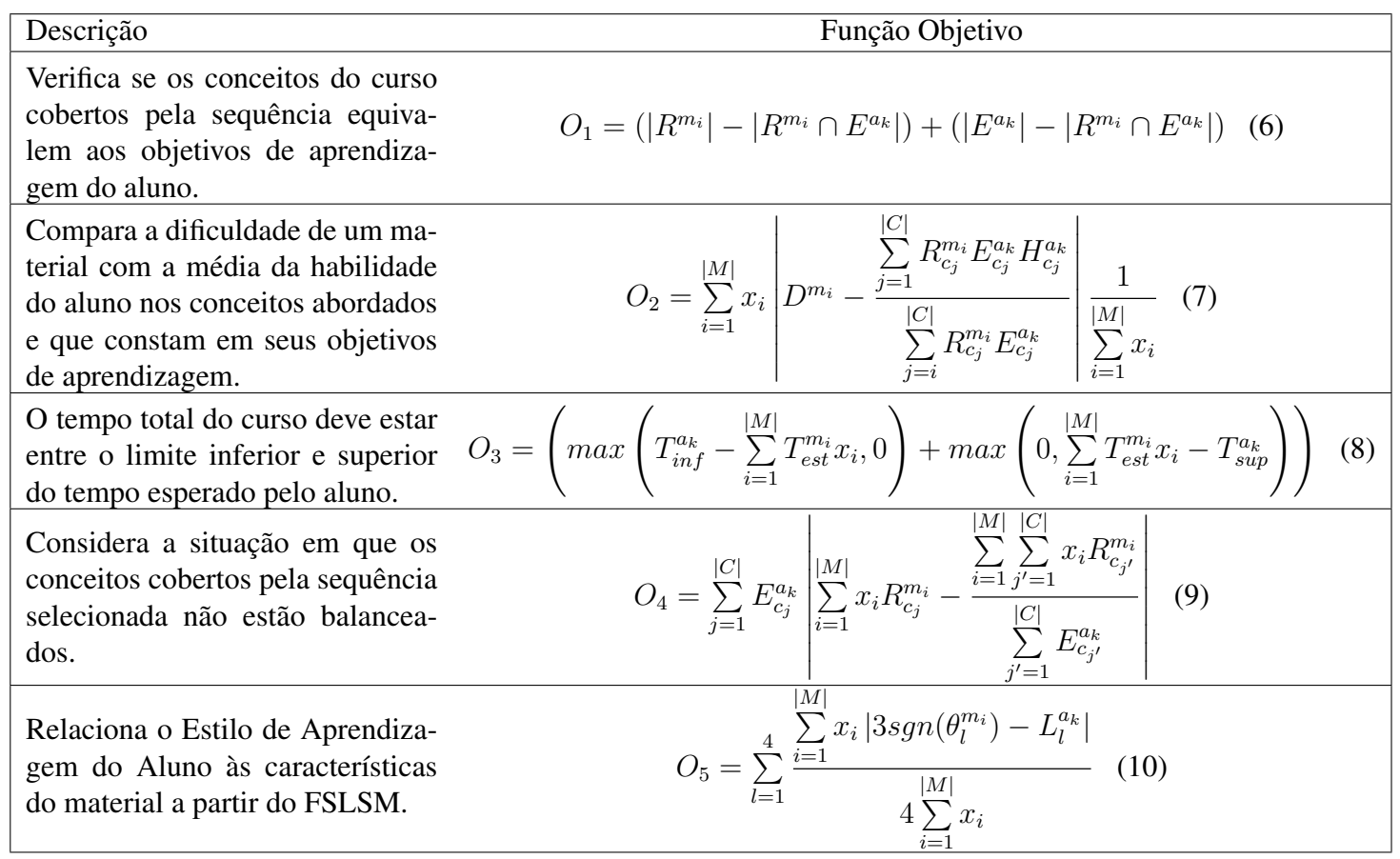

Tabela 1. Funções utilizadas para compor a função objetivo. 
VII Congresso Brasileiro de Informática na Educação (CBIE 2018)

Anais do XXIX Simpósio Brasileiro de Informática na Educação (SBIE 2018)

\section{Resultados e análise}

Para avaliação da proposta, foram criados 3 repositórios $^{2}$ contendo 100, 200 e 300 materiais didáticos, respectivamente, e dados de alunos fictícios. Os materiais nesses repositórios abordam 15 conceitos de um determinado curso. Todos os dados estão de acordo com os parâmetros esperados na função objetivo. Além disso, os dados dos materiais didáticos foram preenchidos utilizando o modelo IEEE-LOM. Variou-se a quantidade de iterações do algoritmo (de 50 em 50 até 450) e o tamanho da população (10, 20 e 30 indivíduos). Nenhum objetivo foi priorizado em relação a outro, isto é, não foram associados pesos $\left(\omega_{i}\right)$ na utilização da Equação 5 Os demais parâmetros foram escolhidos empiricamente: $\lambda_{\min }=4, \lambda_{\max }=\frac{|M|}{4}$ (onde $\mathrm{M}$ representa o conjunto de materiais de um repositório), chance de seguir presas melhores é de $80 \%, \eta=1, \tau=1$ e $\beta=1$. Foram realizadas 10 execuções para cada combinação de parâmetros.

Os resultados apresentados na Tabela 2 demonstram o comportamento do algoritmo através dos melhores (Menores), piores (Maiores) e valores médios encontrados pela variação dos parâmetros: tamanho da população, tamanho do repositório e quantidade de iterações. O cabeçalho $\mathbf{I}$ indica o número de iterações e $\mathbf{P}$ indica o tamanho da população. O gráfico da Figura 2(a) foi criado a partir dos valores Médios e ilustra a convergência do algoritmo a partir do repositório com 300 materiais didáticos. É possível notar uma rápida convergência em direção ao valor mínimo logo nas primeiras 200 iterações onde o valor de fitness diminui quase pela metade a cada 50 iterações.

Não foi possível notar diferença considerável ao aumentar o tamanho da população. Isso pode ser levado em consideração quando o tempo de execução é um fator importante, pois naturalmente esse tempo aumenta a medida que o tamanho da população também aumenta, vide Figura 2(b) ${ }^{3}$.

Pode-se perceber através da Tabela 2 que a quantidade de iterações necessárias para encontrar o menor valor de fitness é impactada pelo tamanho do repositório. Isso se dá porque o espaço de busca é maior, gerando mais combinações. Entretanto, a curva de convergência mantém o comportamento esperado.

\section{Conclusões}

A efetiva utilização dos sistemas de $e$-learning, depende de soluções para os diversos desafios que norteiam a área. Neste trabalho, é apresentada uma solução para o problema de SCA, o qual se mostra como um dos principais desafios na área de aprendizagem adaptativa. O problema foi abordado a partir do Algoritmo Presa-Predador que ainda não havia sido explorado pela literatura de SCA. Buscando considerar os diversos parâmetros dos alunos e da base de conhecimento, a função objetivo utilizada pelo APP é uma agregação de cinco funções, valendo-se ressaltar a função relacionada ao EA, pois essa considera a intensidade de preferência do aluno por um determinado EA do material didático.

A validade da proposta foi demonstrada a partir dos experimentos, onde o APP foi capaz de convergir a partir da função objetivo definida, alcançando os menores valores com poucas iterações. [Tilahun and Ong 2015] indicam como um dos seus trabalhos

\footnotetext{
${ }^{2}$ https://github.com/ufjf-dcc/LAPIC3-benchmark/tree/master/LaboratoryExperiments/Databases

${ }^{3}$ Os experimentos foram realizados em um computador com processador Intel(R) Core(TM) i7-4790 $C P U$ @3.60GHz e 16GB de RAM. Todo o código foi desenvolvido em Java.
} 
VII Congresso Brasileiro de Informática na Educação (CBIE 2018)

Anais do XXIX Simpósio Brasileiro de Informática na Educação (SBIE 2018)

\begin{tabular}{|c|c|c|c|c|c|c|c|c|c|c|}
\hline & \multicolumn{9}{|c|}{ Tamanho do repositório de materiais didáticos } \\
\hline & & \multicolumn{3}{|c|}{100} & \multicolumn{3}{|c|}{200} & \multicolumn{3}{|c|}{300} \\
\hline & & \multicolumn{3}{|c|}{ Valores de fitness } & \multicolumn{3}{|c|}{ Valores de fitness } & \multicolumn{3}{|c|}{ Valores de fitness } \\
\hline I & $\mathrm{P}$ & Menores & Maiores & Médios & Menores & Maiores & Médios & Menores & Maiores & Médios \\
\hline \multirow{3}{*}{50} & 10 & 26,181 & 60,233 & 39,9924 & 167,145 & 225,739 & 196,5059 & 389,866 & 490,696 & 440,5511 \\
\hline & 20 & 27,114 & 66,804 & 45,2313 & 168,164 & 254,266 & 203,26 & 381,963 & 497,357 & 442,3112 \\
\hline & 30 & 29,136 & 69,407 & 42,8237 & 180,543 & 223,147 & 200,5024 & 394,579 & 477,161 & 437,9121 \\
\hline \multirow{3}{*}{100} & 10 & 6,628 & 15,903 & 12,0725 & 68,693 & 114,784 & 84,4226 & 211,12 & 269,166 & 238,7788 \\
\hline & 20 & 6,31 & 19,412 & 11,3411 & 38,25 & 106,099 & 75,0501 & 172,988 & 230,235 & 202,6429 \\
\hline & 30 & 2,687 & 14,378 & 11,1784 & 51,608 & 100,113 & 73,8104 & 156,673 & 271,734 & 218,7392 \\
\hline \multirow{3}{*}{150} & 10 & 6,428 & 19,776 & 10,4356 & 20,737 & 53,123 & 35,6479 & 91,28 & 151,965 & 118,0335 \\
\hline & 20 & 4,187 & 15,299 & 9,144 & 20,3 & 38,546 & 29,7752 & 68,635 & 148,759 & 114,8693 \\
\hline & 30 & 3,027 & 13,218 & 8,3962 & 26,627 & 48,622 & 40,1156 & 88,478 & 145,432 & 113,5729 \\
\hline \multirow{3}{*}{200} & 10 & 2,687 & 13,865 & 7,9839 & 8,739 & 24,503 & 15,8604 & 41,362 & 98,184 & 65,8263 \\
\hline & 20 & 3,027 & 10,958 & 8,3884 & 7,693 & 34,099 & 19,4947 & 48,956 & 92,848 & 64,1679 \\
\hline & 30 & 5,704 & 10,49 & 7,8415 & 7,653 & 20,848 & 13,082 & 25,36 & 84,951 & 62,94 \\
\hline \multirow{3}{*}{250} & 10 & 6,4 & 12,967 & 8,433 & 6,1 & 26,529 & 13,6351 & 22,471 & 60,963 & 40,1279 \\
\hline & 20 & 2,687 & 10,049 & 7,591 & 5,971 & 13,762 & 10,2092 & 17,942 & 45,081 & 35,0715 \\
\hline & 30 & 2,687 & 10,049 & 7,4564 & 5,168 & 17,96 & 9,8317 & 23,92 & 52,739 & 36,9628 \\
\hline \multirow{3}{*}{300} & 10 & 4,187 & 10,899 & 7,4219 & 5,242 & 16,919 & 10,499 & 12,432 & 51,127 & 27,4195 \\
\hline & 20 & 3,187 & 11,152 & 6,9589 & 7,013 & 16,78 & 9,7369 & 19,919 & 49,256 & 27,2218 \\
\hline & 30 & 2,687 & 11,558 & 6,6136 & 7,411 & 14,333 & 9,5389 & 11,447 & 30,396 & 21,1476 \\
\hline \multirow{3}{*}{350} & 10 & 6,746 & 11,435 & 8,1063 & 4,71 & 16,562 & 9,9412 & 9,566 & 21,894 & 16,1912 \\
\hline & 20 & 4,187 & 11,523 & 8,293 & 6,59 & 11,109 & & 7,89 & 29,857 & 16,0991 \\
\hline & 30 & 2,687 & 9,019 & 5,3238 & 4,338 & 15,966 & 8,6576 & 9,079 & 32,883 & 21,3966 \\
\hline \multirow{3}{*}{400} & 10 & 3,027 & 10,386 & 6,2994 & 7,21 & 12,888 & 9,4138 & 8,379 & 22,579 & 14,5969 \\
\hline & 20 & 2,687 & 10,328 & 6,5498 & 5,619 & 19,069 & 9,7943 & 5,533 & 20,824 & 13,5567 \\
\hline & 30 & 2,687 & 11,794 & 7,619 & 6,326 & 14,432 & 9,2658 & 6,278 & 22,786 & 14,9721 \\
\hline \multirow{3}{*}{450} & 10 & 2,687 & 12,278 & 7,2583 & 4,559 & 16,629 & 9,1352 & 7,372 & 23,484 & 14,8671 \\
\hline & 20 & 2,687 & 12,417 & 7,7981 & 5,241 & 11,311 & 7,8156 & 7,783 & 20,896 & 14,0026 \\
\hline & 30 & 5,704 & 9,93 & 7,5727 & 6,679 & 10,742 & 8,754 & 9,324 & 24,997 & 14,8906 \\
\hline
\end{tabular}

Tabela 2. Valores de fitness de cada execução.

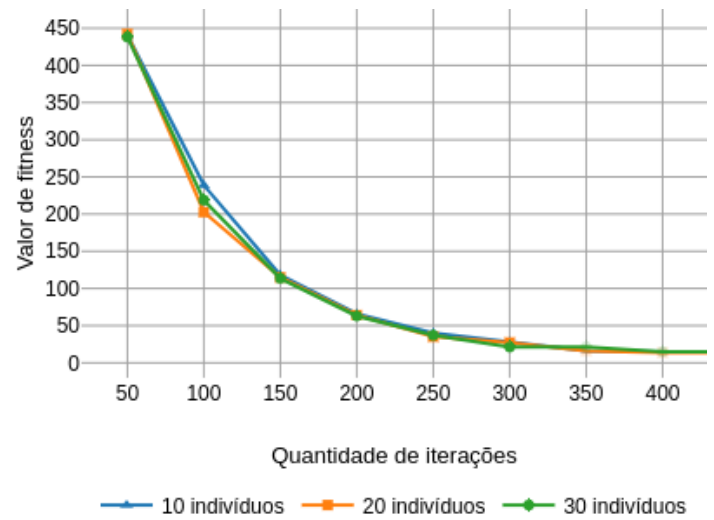

(a) Curva de convergência.

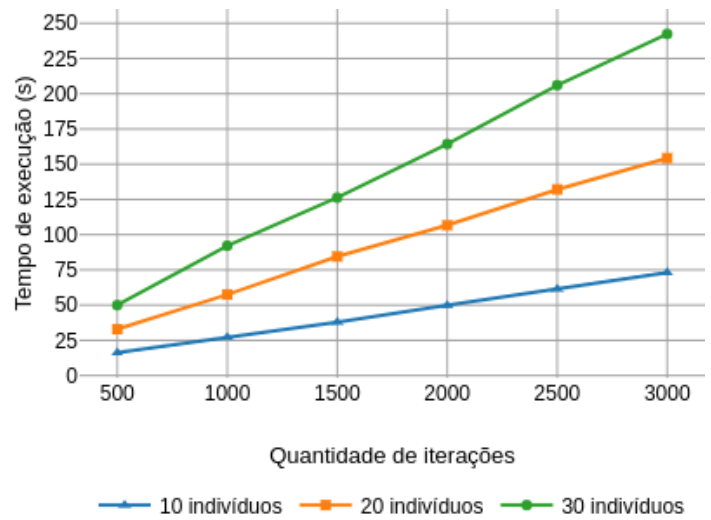

(b) Tempo de execução.

Figura 2. Resultados dos experimentos para repositório com $\mathbf{3 0 0}$ materiais.

futuros a utilização do APP em problemas reais para confirmar a validade da solução. Assim, este trabalho se apresenta como uma contribuição direta. Embora a abordagem não tenha sido comparada com outras meta-heurísticas, a rápida convergência da solução mostra que a abordagem pode ser explorada por pesquisadores para tratar o problema de SCA. Além disso, este trabalho propôs uma modelagem binária para a solução que pode 
VII Congresso Brasileiro de Informática na Educação (CBIE 2018)

Anais do XXIX Simpósio Brasileiro de Informática na Educação (SBIE 2018)

ser estendida para problemas semelhantes.

Para melhor avaliação da proposta, esta abordagem será aplicada em um curso real, de forma a validar a influência do SCA no desempenho final dos alunos. Ainda, é pretendido investigar e incluir novos critérios no processo de otimização, tais como a qualidade de conexão, informações do dispositivo de acesso e habilidades cognitivas dos alunos. Bem como, pretende-se fazer um estudo mais completo dos parâmetros utilizados no APP, buscando melhorar a convergência e desempenho da solução. A variação da solução de acordo com o número de predadores, melhores presas e presas que são seguidas no momento do caminhamento podem gerar melhores resultados. Nesse contexto, será feita a comparação de eficiência em referência a outras meta-heurísticas que foram utilizadas para o mesmo problema,tais como o AG e OEP.

Ademais, devido aos questionamentos sobre a utilização do EA no processo de ensino [Dembo and Howard 2007, Kirschner 2017, Husmann and O'Loughlin 2018], se faz necessário um estudo minucioso para verificar quando esse deve ser completamente descartado ou se há casos passíveis de sua utilização na aprendizagem adaptativa.

\section{Agradecimentos}

O autor Marcelo de Oliveira Costa Machado agradece à CAPES e ao CNPq pelo apoio financeiro concedido a essa pesquisa desenvolvida em seu projeto de Mestrado.

\section{Referências}

Acampora, G., Gaeta, M., and Loia, V. (2011). Hierarchical optimization of personalized experiences for e-learning systems through evolutionary models. Neural Computing and Applications, 20(5):641-657.

Al-Muhaideb, S. and Menai, M. E. B. (2011). Evolutionary computation approaches to the curriculum sequencing problem. Natural Computing, 10(2):891-920.

Brusilovsky, P. (2003). Adaptive and intelligent technologies for web-based education. International Journal of Artificial Intelligence in Education, 13(4):159-172.

Butcher, N. (2015). A basic guide to open educational resources (OER). Commonwealth of Learning (COL);

Dembo, M. H. and Howard, K. (2007). Advice about the use of learning styles: A major myth in education. Journal of college reading and learning, 37(2):101-109.

do Nascimento, P., Barreto, R., Primo, T., Gusmão, T., and Oliveira, E. (2017). Recomendação de objetos de aprendizagem baseada em modelos de estilos de aprendizagem: Uma revisão sistemática da literatura. In Simpósio Brasileiro de Informática na Educação, volume 28, page 213.

Felder, R. M., Silverman, L. K., et al. (1988). Learning and teaching styles in engineering education. Engineering education, 78(7):674-681.

Hafidi, M. and Bensebaa, T. (2015). Architecture for an adaptive and intelligent tutoring system that considers the learner's multiple intelligences. International Journal of Distance Education Technologies, 13(1):1-21. 
VII Congresso Brasileiro de Informática na Educação (CBIE 2018)

Anais do XXIX Simpósio Brasileiro de Informática na Educação (SBIE 2018)

Hnida, M., Idrissi, M. K., and Bennani, S. (2016). Adaptive teaching learning sequence based on instructional design and evolutionary computation. In International Conference on Information Technology Based Higher Education and Training, pages 1-6.

Husmann, P. R. and O'Loughlin, V. D. (2018). Another nail in the coffin for learning styles? disparities among undergraduate anatomy students' study strategies, class performance, and reported vark learning styles. Anatomical sciences education.

Kardan, A. A., Aziz, M., and Shahpasand, M. (2015). Adaptive systems: a content analysis on technical side for e-learning environments. Artificial Intelligence Review, 44(3):365-391.

Kirschner, P. A. (2017). Stop propagating the learning styles myth. Computers \& Education, 106:166-171.

Li, J.-W., Chang, Y.-C., Chu, C.-P., and Tsai, C.-C. (2012). A self-adjusting e-course generation process for personalized learning. Expert Systems with Applications, 39(3):3223-3232.

Machado, M., Souza, J., and Barrére, E. (2017). Geração de sequências curriculares adaptativas baseada em computação evolucionária: Estado da arte e tendências. volume 28, page 1137.

Muhammad, A., Zhou, Q., Beydoun, G., Xu, D., and Shen, J. (2016). Learning path adaptation in online learning systems. In International Conference on Computer Supported Cooperative Work in Design, pages 421-426. IEEE.

Nwana, H. S. (1990). Intelligent tutoring systems: an overview. Artificial Intelligence Review, 4(4):251-277.

Phobun, P. and Vicheanpanya, J. (2010). Adaptive intelligent tutoring systems for elearning systems. Procedia-Social and Behavioral Sciences, 2(2):4064-4069.

Premlatha, K. and Geetha, T. (2015). Learning content design and learner adaptation for adaptive e-learning environment: a survey. Artificial Intelligence Review, 44(4):443465 .

Pushpa, M. (2012). Aco in e-learning: Towards an adaptive learning path. International Journal on Computer Science and Engineering, 4(3):458.

Silva, R. C., Direne, A. I., Marczal, D., Borille, A. C., Guimarães, P. R. B., da Silva Cabral, A., and Camargo, B. F. (2018). Adaptability of learning objects using calibration and adaptive sequencing of exercises. Brazilian Journal of Computers in Education, 26(01):70.

Tilahun, S. L. and Ong, H. C. (2015). Prey-predator algorithm: A new metaheuristic algorithm for optimization problems. International Journal of Information Technology \& Decision Making, 14(06):1331-1352.

Van Merriënboer, J. J. and Ayres, P. (2005). Research on cognitive load theory and its design implications for e-learning. Educational Technology Research and Development, 53(3):5-13.

Xie, H., Zou, D., Wang, F. L., Wong, T.-L., Rao, Y., and Wang, S. H. (2017). Discover learning path for group users: A profile-based approach. Neurocomputing, 254:59-70. 